the integral of $1 / \sqrt{x}+1 / \sqrt{y}+1 / \sqrt{z}$, was approximated over the cube with center $(1 / 2,1 / 2,1 / 2)$ and edge 1 . The 34-point formula here described was used and the result was 6.04 compared to the exact value 6 .

Conclusion. We have here summarized a few of the useful theorems for generating numerical integration formulas from given ones, and illustrated one particular approach to obtaining formulas for symmetrical regions. In a sequel we will present all the particular formulas we have calculated for symmetrical regions including some for circle, square, sphere, cube, hypersphere, and hypercube. The method of attack presented for symmetrical regions does not hold much promise of effective extension to arbitrarily high-degree polynomials such as Peirce has carried out for the circular annulus. However, variations and extensions of these methods will be forthcoming.

Preston C. Hammer

A. WAYNE WYMORE

University of Wisconsin

Madison, Wisconsin

This work is supported by a grant of Wisconsin Alumni Research Foundation funds made by the Graduate Research Committee, and by Office of Ordnance Research, U. S. Army contract no. DA-11-022-ORD-2301.

1. J. Clerk-Maxwell, "On approximate multiple integration between limits of summation," Cambridge Phil. Soc., Proc., v. 3, 1877, p. 39-47.

2. G. W. TYLER, "Numerical integration of functions of several variables," Canadian Jn. Math., v. 5, 1953 , p. 393-412.

3. P. C. Hammer, O. J. Marlowe \& A. H. Stroud, "Numerical integration over simplexes and cones," $M T A C$, v. 10, 1956, 130-137.

4. P. C. Hammer, \& A. H. Stroud, "Numerical integration over simplexes," $M T A C$, v. 10, 1956, p. $137-139$.

5. W. H. PEIRCE, "Numerical integration over planar regions," Ph.D. Thesis, University of Wisconsin, 1956, available on microfilm.

6. P. Davis \& P. Rabinowitz, "Some Monte Carlo experiments in computing multiple integrals," $M T A C$, v. 10, 1956, p. 1-8.

\title{
Solution of Poisson's Equation by Relaxation Method-Normal Gradient Specified on Curved Boundaries
}

Introduction. In the application of the relaxation technique to the solution of the Poisson's equation when the normal gradient of the wanted function, $w$, is specified along a curved boundary, difficulty has been experienced in finding a suitable finite difference approximation to the Poisson's equation at nodes that lie adjacent to the curved boundary. It is proposed to present in this article results which will be found adequate for tackling any type of curved boundary in two dimensions.

This problem was treated by $R$. V. Southwell by means of the membrane analogy developed by him [1]. It is, however, preferable to base the approximate representation of differential equations on finite difference theory, which indicates the order of the error involved in the approximation. This has been fully achieved in the case of the Poisson's equation when the boundary condition consists in 
(i) the wanted function, $w$, assuming a given value on a boundary of any shape, or

(ii) the normal gradient of the wanted function assuming a given value on a rectangular boundary or a boundary made up of straight lines parallel or perpendicular to the mesh lines.

The general problem of a curved boundary with the normal gradient of $w$ specified thereon, however, has exhibited great technical difficulties in the work of L. Fox [2], for example. Nodes in the diagonal directions are introduced into the approximate formulae by D. N. de G. Allen [3] in order to tackle this problem. In the present treatment approximate formulae are derived by taking into account the rate of change of the normal gradient of $w$ along the boundary.

The formulae developed here lead to simple relaxation patterns. The coefficients occurring in these formulae depend on the curvature of the boundary at the successive nodes. Their computation at the outset necessarily involves heavy arithmetic: The final result can be expected to be correspondingly nearer the

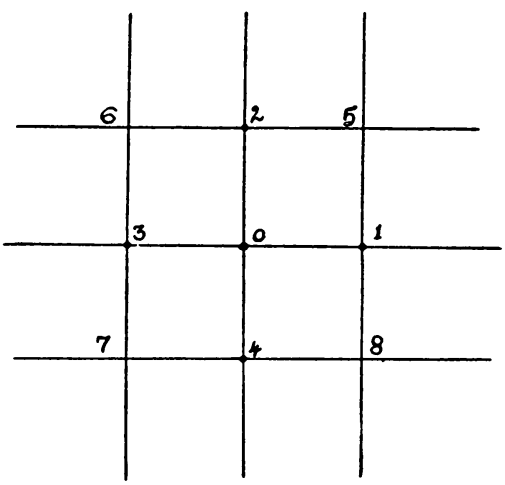

FIG. 1. Neighbouring nodes numbered with reference to Node ' 0 '.

correct solution. Close adherence to the specified boundary condition is ensured as both the curvature of the boundary and the rate of change of the normal gradient of $w$ along the boundary are taken into account by the formulae developed here.

\section{Symbols and Notation}

$w \quad$ the function which is to be evaluated in a given region

$W \quad$ a function given in the region of integration

$k \quad$ a function given on the boundary of the region of integration

$\nabla^{2} \quad$ the Laplacian operator

0 the node at which a residual formula is required

$F_{0} \quad$ residual at 0

$1,2, \ldots, 8$ Neighbouring nodes that surround 0 ; these are to be identified according to the scheme shown in Fig. 1

$x, y \quad$ Cartesian co-ordinates with respect to suitable axes of reference. The position of the origin and the orientation of the axes are different in different contexts, and are defined where required 
$h: \quad$ mesh size

$\rho: \quad$ radius of curvature with the proper sign, plus or minus

$s: \quad$ distance measured along the boundary

$\alpha: \quad$ an angle defined as in Fig. 4 or Fig. 5

$\xi$ : $\quad$ a ratio defined as in Fig. 4 or Fig. 5

$m: \quad \tan \alpha$.

The Problem. Determine the value of a function $w$ inside a region $R$ given that

$$
\begin{aligned}
\nabla^{2} w & =W \quad \text { a given function, at points inside } R, \\
\partial w / \partial \nu & =k \quad \text { on the boundary of } R .
\end{aligned}
$$

Here $\partial / \partial \nu$ denotes the rate of change along the outward-drawn normal to the boundary, and $k$ is a function given along the boundary.

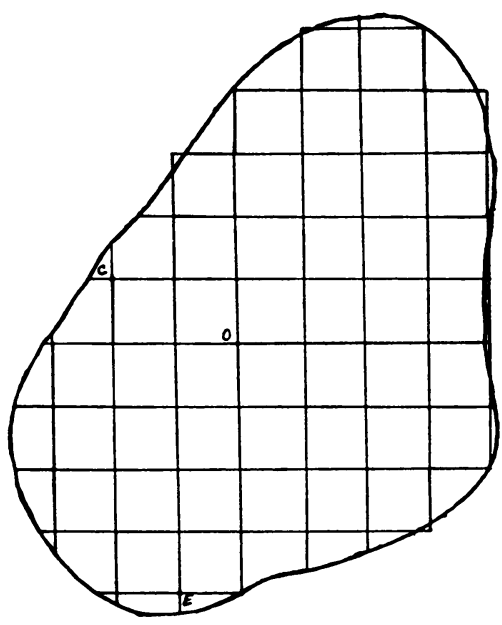

FIG. 2

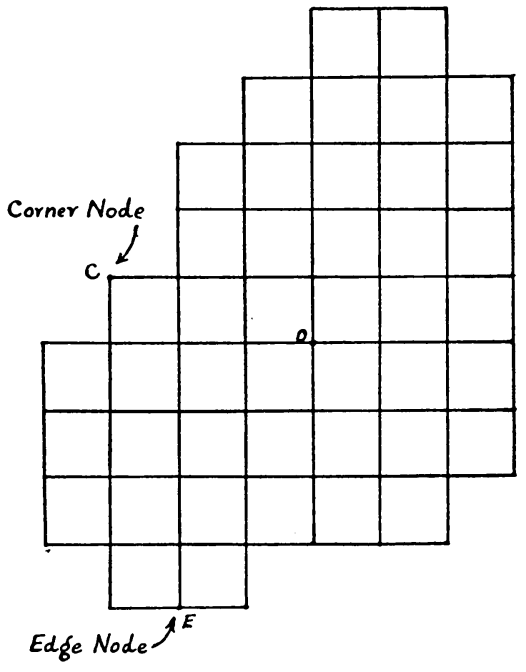

FIG. 3

Network and Types of Nodes. For solving the above problem, the region will be covered by a network of square meshes of suitable mesh size as shown in Fig. 2, and the value of $w$ will be ascertained at the nodes of this network by the method of relaxation. The diagram of the network is shown separately in Fig. 3, as this diagram will engage our attention throughout the process of relaxation. On examining this diagram, we find that there are three types of nodes in the network.

(a) For most of the nodes (i.e., nodes not belonging to the two types discussed below) there are four neighbouring nodes, which can be numbered $1,2,3$, and 4 in accordance with the scheme set out in Fig. 1. These nodes will be called "interior nodes."

(b) For nodes which occur on the edge of the network, one of the neighbouring nodes $1,2,3$, or 4 is missing ; e.g., node $E$ for which there is no neighbour which can be marked with the number 4 . Such nodes will be called "edge nodes." 
(c) For nodes which occur at a corner of the network two of the neighbouring nodes are missing; e.g., node $C$ for which there are no neighbours which can be marked 2 or 3 . These nodes will be called "corner nodes."

In the case of an interior node it is easily derived [3] that the finite difference approximation to the Poisson's equation is

$$
h^{2} W_{0}=w_{1}+w_{2}+w_{3}+w_{4}-4 w_{0}+O\left(h^{4}\right),
$$

where the suffixes denote the nodes at which the values of the concerned functions are to be taken. This result is obtained on assuming that the wanted function possesses a Taylor's series at the point $O$, with a circle of convergence large enough to enclose nodes $1,2,3$, and 4 .

The above approximation is of no avail at the edge and corner nodes, as at these nodes one or two of the neighbouring nodes, $1,2,3$, and 4 , are missing. The boundary condition which $w$ has to satisfy, has to be utilized in order to eliminate the values of $w$ at the missing nodes from equation (3). This is achieved as follows.

Edge node. Taking an edge node 0 let it be assumed that node 1 is missing (Fig. 4). Let it be assumed, again, that the wanted function, $w$, possesses a

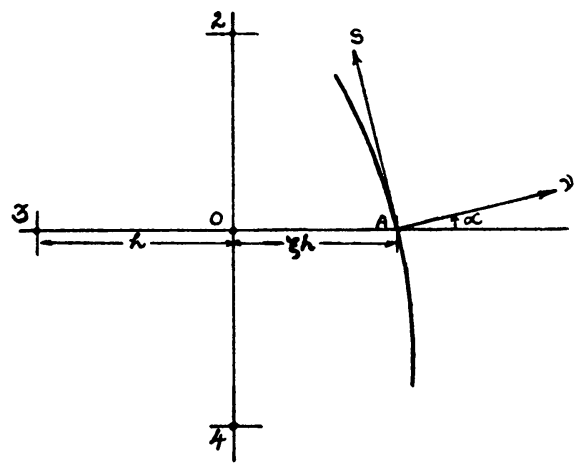

FIG. 4

Taylor's series at 0 , with a circle of convergence large enough to enclose nodes 2 , 3 , and 4.

Let the series be

$$
\begin{aligned}
w=w_{0}+p x+q y+b x^{2}+2 f x y & +c y^{2} \\
& +a_{1} x^{3}+3 b_{1} x^{2} y+3 c_{1} x y^{2}+d_{1} y^{3}+\cdots
\end{aligned}
$$

where $x, y$ are cartesian co-ordinates with origin at 0 and $x$-axis along the straight line 3-0-1.

Since $\nabla^{2} w=W$ at any point in $R$,

$$
2(b+c)=W_{0} .
$$

Let the straight line, $3-0-1$ cut the boundary at $A$. The distance $O A$ is less than or equal to $h$. Let $O A=\xi h$, where $0 \leq \xi \leq 1$ (vide Fig. 4). Let the normal at $A$ 
to the boundary make an angle $\alpha$ with the $x$-axis (i.e., 3-0-1), in the anticlockwise direction.

The given boundary condition states that

$$
(\partial w / \partial \nu)_{A}=k_{A}
$$

Since $\partial w / \partial \nu=k$ all along the boundary, it can be derived that

$$
\left(\partial^{2} w / \partial s \partial \nu\right)_{A}=(d k / d s)_{A},
$$

on assuming that $k$ possesses a derivative along the boundary at $A$. $[\partial / \partial$ s denotes the rate of change along the boundary.]

It is easy to express $(\partial w / \partial \nu)_{A}$ and $\left(\partial^{2} w / \partial s \partial \nu\right)_{A}$ in terms of the coefficients in the expansion (4) since along the curve,

$$
\partial w / \partial \nu=\partial w / \partial x \cos (x, \nu)+\partial w / \partial y \cos (y, \nu)
$$

and

(9) $\partial^{2} w / \partial s \partial \nu=\partial^{2} w / \partial x \partial y\left[\cos ^{2}(x, \nu)-\cos ^{2}(y, \nu)\right]$

$$
\begin{aligned}
+\left[\partial^{2} w / \partial y^{2}-\partial^{2} w / \partial x^{2}\right] \cos (x, \nu) \cos (y, \nu) \\
-1 / \rho[\partial w / \partial x \cos (y, \nu)-\partial w / \partial y \cos (x, \nu)] .
\end{aligned}
$$

Here $\rho$ is the radius of curvature given by $d s / d \psi$ in the usual notation. The symbols $(x, \nu)$ and $(y, \nu)$ indicate the angles between the $x$ - and the $\nu$-directions, and the $y$ - and the $\nu$-directions, respectively.

By differentiating the series (4) successively and substituting $x=\xi h, y=0$; in the results, the following approximate values are obtained at the point $A$.

$$
\begin{aligned}
(\partial w / \partial x)_{A} & =p+2 b \xi h+O\left(h^{2}\right), \\
(\partial w / \partial y)_{A} & =q+2 f \xi h+O\left(h^{2}\right), \\
\left(\partial^{2} w / \partial x^{2}\right)_{A} & =2 b+O(h), \\
\left(\partial^{2} w / \partial x \partial y\right)_{A} & =2 f+O(h),
\end{aligned}
$$

and

$$
\left(\partial^{2} w / \partial y^{2}\right)_{A}=2 c+O(h) .
$$

Combining (6), (8), (10), and (11) we obtain

$$
k_{A}=p \cos \alpha+q \sin \alpha+2 \xi h(b \cos \alpha+f \sin \alpha)+O\left(h^{2}\right) .
$$

Similarly combining (7), (9), and (10) through (14) we obtain

[B] $\left(\frac{d k}{d s}\right)_{A}=-p \frac{\sin \alpha}{\rho_{A}}+q \frac{\cos \alpha}{\rho_{A}}+2\{f \cos 2 \alpha+\overline{c-b} \sin \alpha \cos \alpha\}+O(h)$. 
On multiplying [A] by $\sec \alpha$ and [B] by $\xi h \tan \alpha \sec 2 \alpha$ and taking the difference,

(15) $k_{A} \sec \alpha-\xi h \tan \alpha \sec 2 \alpha(d k / d s)_{A}$

$=p\left(1+\xi h \sin \alpha \tan \alpha \sec 2 \alpha / \rho_{A}\right)+q\left(\tan \alpha-\xi h \sin \alpha \sec 2 \alpha / \rho_{A}\right)$

$+2 b \xi h-(c-b) \xi h \tan \alpha \tan 2 \alpha+O\left(h^{2}\right)$.

On substituting the co-ordinates $(0, h),(-h, 0)$ and $(0,-h)$ respectively in the series (4), the values of $w_{2}, w_{3}$, and $w_{4}$ are obtained. From these it can be deduced that

$$
\begin{aligned}
w_{2}+w_{4}-2 w_{0} & =2 c h^{2}+O\left(h^{4}\right) \\
w_{2}-w_{4} & =2 h q+O\left(h^{3}\right)
\end{aligned}
$$

and

$$
w_{3}-w_{0}=-p h+b h^{2}+O\left(h^{3}\right) .
$$

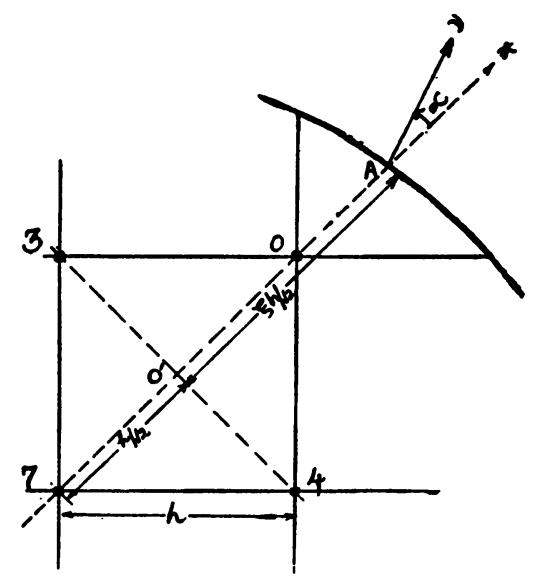

Fig. 5

Combining (5), (15), and (16) to eliminate the unknown coefficients, we obtain an elegant result,

$$
\begin{aligned}
W_{0} h^{2}(1+ & \left.+2 \xi \frac{1}{1-m^{2}}\right)-2 h k_{A} \sqrt{1+m^{2}}+2\left(\frac{d k}{d s}\right)_{A} \xi h^{2} m \frac{1+m^{2}}{1-m^{2}} \\
= & w_{2}\left(1-m+2 \xi \frac{1+m^{2}}{1-m^{2}}+\xi \frac{h}{\rho_{A}} \frac{m \sqrt{1+m^{2}}}{1-m}\right) \\
& +2 w_{3}\left(1+\xi \frac{h}{\rho_{A}} \frac{m^{2} \sqrt{1+m^{2}}}{1-m^{2}}\right) \\
& +w_{4}\left(1+m+2 \xi \frac{1+m^{2}}{1-m^{2}}-\xi \frac{h}{\rho_{A}} \frac{m \sqrt{1+m^{2}}}{1+m}\right) \\
& -4 w_{0}\left(1+\xi \frac{1+m^{2}}{1-m^{2}}+\xi \frac{h}{\rho_{A}} \frac{m^{2} \sqrt{1+m^{2}}}{1-m^{2}}\right)+O\left(h^{3}\right)
\end{aligned}
$$

where $m=\tan \alpha$. 
The first member of this equation can be calculated at every one of the edge nodes, as soon as a suitable square net is fitted to the region of integration, since this expression depends only on the given functions $W$ and $k$, and the characteristics of the boundary curve. Similarly all the coefficients of the $w$ on the right hand side can be determined at the outset.

Corner node. The method of obtaining a residual formula at a corner node involves a few geometrical constructions, which are indicated in Fig. 5 for the case in which nodes 1 and 2 are missing. Taking our stand for a moment at $0^{\prime}$ in Fig. 5, we can derive as we have done above, that

$$
\begin{aligned}
W_{0}, \frac{h^{2}}{2} & {\left[1+2 \xi \frac{1}{1-m^{2}}\right]-\sqrt{2} h k_{A} \sqrt{1+m^{2}}+\left(\frac{d k}{d s}\right)_{A} \xi h^{2} m \frac{1+m^{2}}{1-m^{2}} } \\
= & w_{3}\left(1-m+2 \xi \frac{1+m^{2}}{1-m^{2}}+\xi \frac{h}{\sqrt{2} \rho_{A}} \cdot \frac{m \sqrt{1+m^{2}}}{1-m}\right) \\
& +2 w_{7}\left(1+\xi \frac{h}{\sqrt{2} \rho_{A}} \frac{m^{2} \sqrt{1+m^{2}}}{1-m^{2}}\right) \\
& +w_{4}\left(1+m+2 \xi \frac{1+m^{2}}{1-m^{2}}-\xi \frac{h}{\sqrt{2} \rho_{A}} \frac{m \sqrt{1+m^{2}}}{1+m}\right) \\
& -4 w_{0},\left(1+\xi \frac{1+m^{2}}{1-m^{2}}+\xi \frac{h}{\sqrt{2} \rho_{A}} \cdot m^{2} \cdot \frac{\sqrt{1+m^{2}}}{1-m^{2}}\right)+O\left(h^{3}\right) .
\end{aligned}
$$

The positions of $0^{\prime}$ and $A$ are to be noted carefully. Again

$$
0^{\prime}-3=0^{\prime}-7=0^{\prime}-4=h / \sqrt{2} \text {. }
$$

Hence (18) is obtained from (17) by merely replacing $h$ by $h / \sqrt{2}$ and giving suffixes to $w$ in accordance with Fig. 5 . It is to be noted that $\xi>1$ here.

Because of Poisson's equation,

$$
\frac{h^{2}}{2} W_{0^{\prime}}=w_{0}+w_{3}+w_{7}+w_{4}-4 w_{0^{\prime}}+O\left(h^{4}\right) .
$$

On eliminating $w_{0}$, between (18) and (19) we obtain

$$
\begin{aligned}
W_{0}, \frac{h^{2}}{2} \xi & -\sqrt{2} h k_{A} \sqrt{1+m^{2}}+\left(\frac{d k}{d s}\right)_{A} \xi h^{2} m \frac{1+m^{2}}{1-m^{2}} \\
= & w_{3}\left(-m+\xi \frac{1+m^{2}}{1-m^{2}}+\frac{\xi h}{\sqrt{2} \rho_{A}} \frac{m \sqrt{1+m^{2}}}{1-m^{2}}\right) \\
& +w_{7}\left(1-\xi \frac{1+m^{2}}{1-m^{2}}+\frac{\xi h}{\sqrt{2} \rho_{A}} \cdot m^{2} \cdot \frac{\sqrt{1+m^{2}}}{1-m^{2}}\right) \\
& +w_{4}\left(m+\xi \frac{1+m^{2}}{1-m^{2}}-\frac{\xi h}{\sqrt{2} \rho_{A}} \frac{m \sqrt{1+m^{2}}}{1-m^{2}}\right) \\
& -w_{0}\left(1+\xi \frac{1+m^{2}}{1-m^{2}}+\frac{\xi h}{\sqrt{2} \rho_{A}} \cdot \frac{m^{2} \sqrt{1+m^{2}}}{1-m^{2}}\right)+O\left(h^{3}\right) .
\end{aligned}
$$




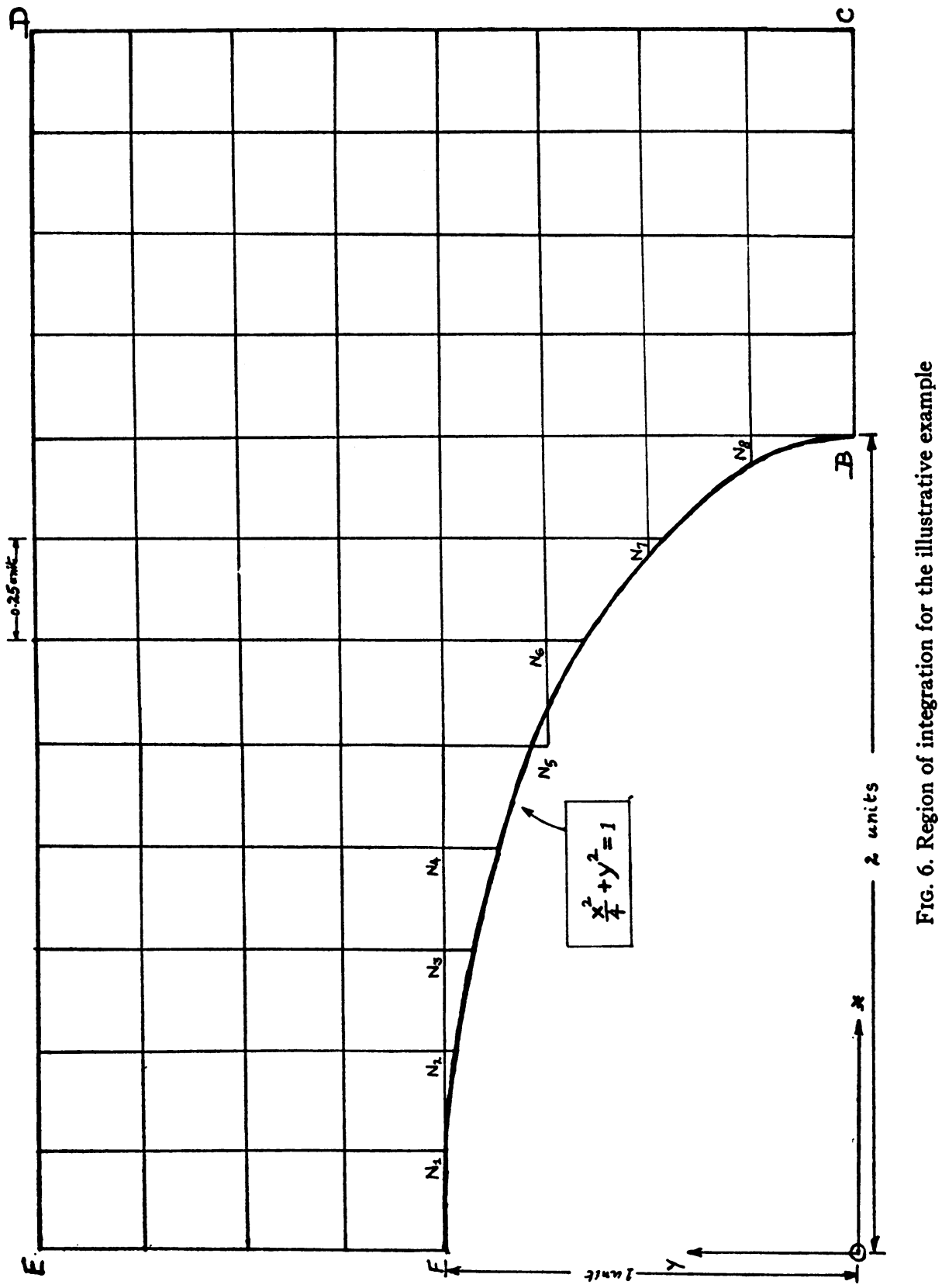




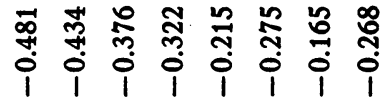

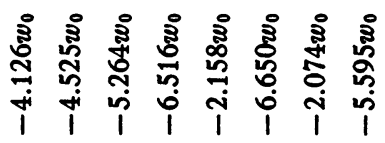

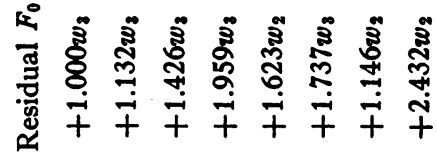

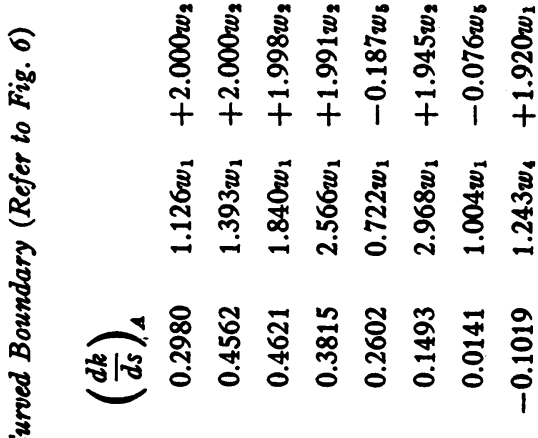

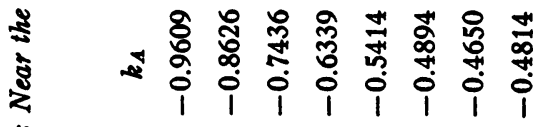

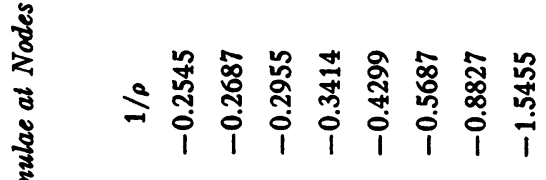

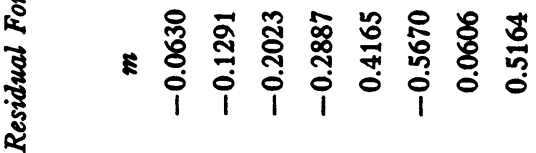

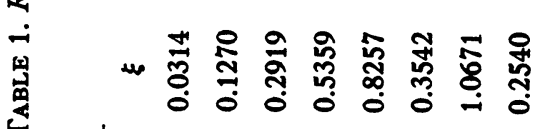

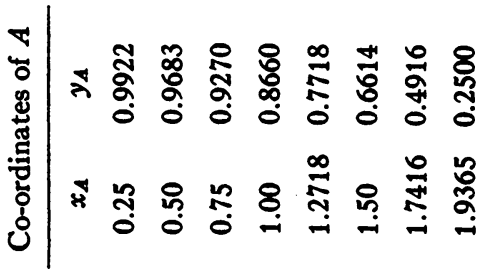

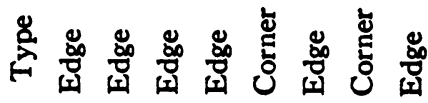

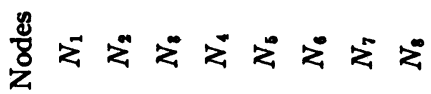


The important points about this formula are:

(i) The value of $W$ to be taken is the value at $0^{\prime}$ and not that at 0 .

(ii) A change in the assumed value of $w$ at node 7 will affect the residual at 0 but not vice versa.

Illustrative Example. The above formulae were applied to calculate the values of $w=\log r=\frac{1}{2} \log \left(x^{2}+y^{2}\right)$ within the region $B C D E F B$ shown in Fig. 6, assuming $\partial w / \partial \nu$ to be given all along the boundary. It is well known that $\log r$ satisfies Laplace's equation in any region of the plane, provided the origin is outside the region. Hence $W \equiv 0$ in equation (1).

The curved boundary $F B$ is part of the ellipse

$$
x^{2} / 4+y^{2}=1 \text {. }
$$

$B C$ and $E F$ lie along the $x$ - and $y$-axes respectively, while $C D$ lies along the line $x=3$, and $D E$ along the line $y=2$.

The network adopted is rather coarse with $h=0.25$ only. There are eight nodes besides $F$ and $B$, which lie adjacent to the elliptic boundary. They are numbered in order in Fig. 6. Of these nodes, $N_{5}$ and $N_{7}$ are corner nodes and the rest, edge nodes. Hence equations (17) and (20) are directly applied to these nodes. Table 1 summarizes all the data required at these nodes for calculating the coefficients in equations (17) or (20), and also gives the corresponding residual formulae.

\section{TABLE 2}

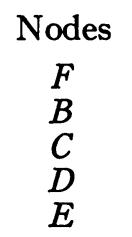

$$
\begin{gathered}
\text { Residual } \\
F_{0}=w_{1}+w_{2}-0.250 \\
F_{0}=w_{1}+w_{2}-0.125 \\
F_{0}=w_{2}+w_{3}+0.083 \\
F_{0}=w_{3}+w_{4}+0.096 \\
F_{0}=w_{4}+w_{1}+0.125
\end{gathered}
$$

At nodes $F$ and $B$ the missing nodes are eliminated from equation (3) by making use of the values $\partial w / \partial x$ and $\partial w / \partial y$ which are both known there. The same is the case at nodes $C, D$, and $E$. The residual formulae for these nodes are given in Table 2.

At all intermediate nodes on $B C, C D, D E$, and $E F$, one of the surrounding nodes is missing and this is eliminated from (3) as either $\partial w / \partial y$ or $\partial w / \partial x$ is known at these nodes. (Alternatively the same result can be obtained from equation (17) by setting $\xi=m=1 / \rho=0$.) The residual formula at a node on $B C$, for example, will take the form

$$
F_{0}=w_{1}+2 w_{2}+w_{3}-4 w_{0}+2 h k_{0}=O\left(h^{3}\right) .
$$

It may be noted that the problem as set up so far is indeterminate in the sense that any constant value can be added to a solution that satisfies the conditions of the problem. For

$$
\begin{aligned}
& \text { if } \quad \nabla^{2} w=W \text { inside a region } R, \\
& \text { and } \partial w / \partial \nu=k \text { on the boundary of } R \text {, }
\end{aligned}
$$


it is clear that

$$
\nabla^{2}(w+c)=W \text { inside } R
$$

and

$$
\partial / \partial \nu(w+c)=k \text { on the boundary, }
$$

where $c$ has the same value at all points. This property is reproduced in the set of residual formulae derived above. Every one of these formulae has the feature

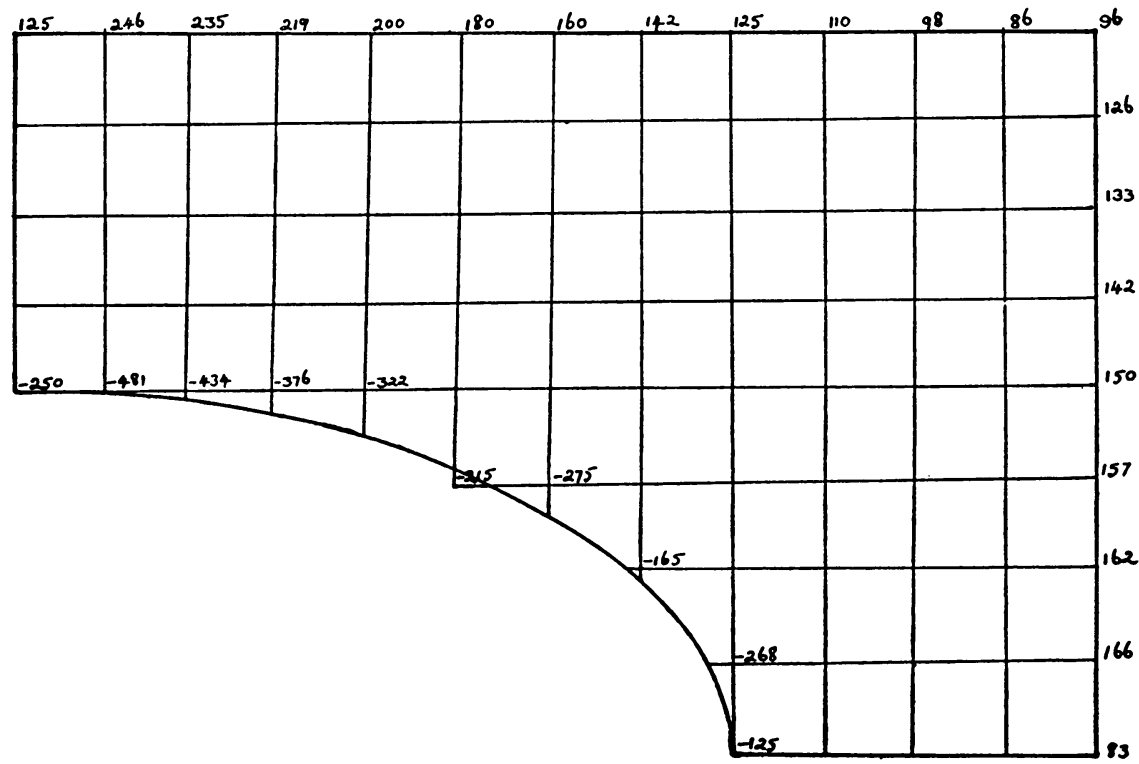

FIG. 6A. The Start: $w$ assumed zero at all nodes. The non-zero residuals multiplied by $10^{3}$ are shown here

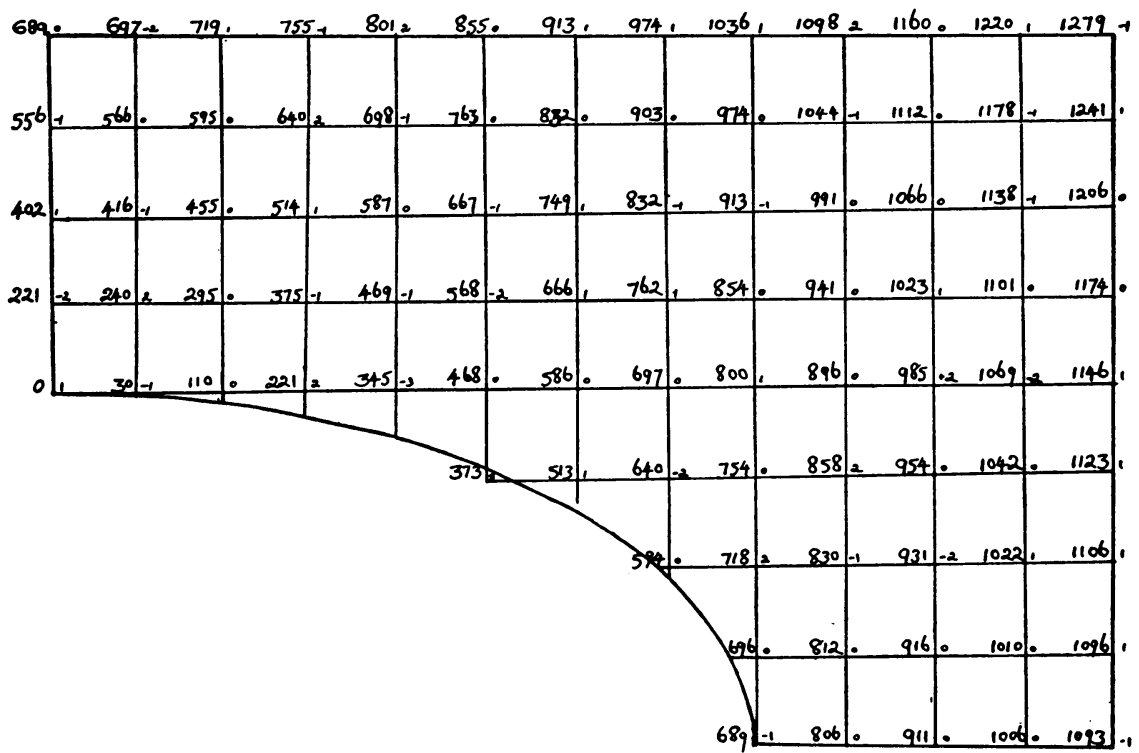

Fig. 7. The End: Values of $w$ multiplied by $10^{3}$ are shown. Final residuals are given 
that the sum of the coefficients of the unknown $w$ is zero. Hence if a set of values of $w$ give rise to a certain set of residuals, then the set of values $(w+c)$ where $c$ is the same for all nodes, will also give rise to the same set of residuals. It is, therefore, necessary to give the value of $w$ at some point in order to make the solution unique. We make use of the fact that $\log r=0$ at $F$ for this purpose.

Starting with the initial guess $w=0$ at all nodes, the solution exhibited in Fig. 7 was obtained by the method of relaxation. The initial residuals are shown in Fig. 6A. During the process of relaxation of the residuals, negative values were obtained for $w$ at nodes like $F, N_{1}$, etc. (vide, Fig. 6 ). When all the residuals had become negligible, a suitable value was added at all the nodes so as to bring the value of $w$ at $F$ to zero.

The values of $\log r$ for select values of $r$ as taken from Fig. 7 are compared in Table 3 with the true values taken from a standard table of Mathematical

TABLE 3

\begin{tabular}{|c|c|c|c|c|c|c|}
\hline \multirow[b]{2}{*}{$r$} & \multicolumn{2}{|c|}{$\log r$} & \multirow[b]{2}{*}{ Node } & \multirow[b]{2}{*}{$r$} & \multicolumn{2}{|c|}{$\log r$} \\
\hline & $\begin{array}{l}\text { by re- } \\
\text { laxation }\end{array}$ & $\begin{array}{c}\text { true } \\
\text { value }\end{array}$ & & & $\begin{array}{l}\text { by re- } \\
\text { laxation }\end{array}$ & $\begin{array}{c}\text { true } \\
\text { value }\end{array}$ \\
\hline 1.00 & 0.000 & 0.000 & $N_{1}$ & 1.0308 & 0.030 & 0.030 \\
\hline 1.25 & 0.221 & 0.223 & $N_{2}$ & 1.1180 & 0.110 & 0.111 \\
\hline 1.50 & 0.402 & 0.405 & $N_{3}$ & 1.2500 & 0.221 & 0.223 \\
\hline 1.75 & 0.556 & 0.560 & $N_{4}$ & 1.4142 & 0.345 & 0.347 \\
\hline 2.00 & 0.689 & 0.693 & $N_{\text {与 }}$ & 1.4577 & 0.373 & 0.377 \\
\hline 2.25 & 0.806 & 0.811 & $N_{6}$ & 1.6771 & 0.513 & 0.517 \\
\hline 2.50 & 0.911 & 0.916 & $N_{7}$ & 1.8200 & 0.594 & 0.599 \\
\hline 2.75 & 1.006 & 1.012 & $N_{8}$ & 2.0156 & 0.696 & 0.701 \\
\hline 3.00 & 1.093 & 1.099 & $D$ & $\sqrt{13.0000}$ & 1.279 & 1.282 \\
\hline
\end{tabular}

Functions, see F. Castle [4]. The agreement is remarkable in spite of the network being coarse.

The author is indebted to D. N. de G. Allen of the Imperial College of Science, London, for the interest shown by him in the publication of this article, when he visited the University of Roorkee, India.

Messrs. C. M. Ganesan and G. Natarajan have been of valuable help to the author in the preparation of the typescript.

\section{R. V. VISWANATHAN}

Central Water and Power Commission

Government of India

New Delhi, India

1. R. V. Southwell, Relaxation Methods in Theoretical Physics, Oxford, at the Clarendon Press, 1946.

2. L. Fox, "Numerical solution of elliptic differential equations when the boundary conditions involve a derivative," Roy. Soc., Phil. Trans., London, v. 242, 1950, p. 345-378.

3. D. N. DE G. Allen, Relaxation Methods, McGraw Hill Book Co., Inc., New York, 1954.

4. F. CASTLE, Five-Figure Logarithmic and Other Tables, Macmillan and Co., London, 1910. 